\title{
28 Research Square \\ Selection and implementation of SNP markers for parentage analysis in a Chinese crossbred cattle population
}

\section{Lirong $\mathrm{Hu}$}

China Agricultural University

\section{Dong Li}

China Agricultural University

\section{Qin Chu}

Beijing Academy of Agriculture and Forestry Science

Yachun Wang ( $\nabla$ wangyachun@cau.edu.cn )

China Agricultural University https://orcid.org/0000-0003-3629-2802

\section{Lei Zhou}

China Agricultural University

\section{Ying Yu}

China Agricultural University

\section{Yuan Zhang}

China Agricultural Universuty

\section{Shengli Zhang}

China Agricultural University

\section{Tahir Usman}

Abdul Wali Khan University

\section{Zhenquan Xie}

Anshan Hengli Dairy Farm

\section{Shiyu Hou}

Anshan Hengli Dairy Farm

Lin Liu

Beijing Dairy Cattle Center

\section{Wanhai Shi}

Beijing Dairy Cattle Center

\section{Research article}

Keywords: Parentage analysis, Single nucleotide polymorphism (SNP), Chinese crossbred cattle 
Posted Date: May 24th, 2020

DOI: https://doi.org/10.21203/rs.3.rs-30446/v1

License: (c) (i) This work is licensed under a Creative Commons Attribution 4.0 International License. Read Full License 


\section{Abstract}

Background: In China, the widespread crossbreeding between Simmental and Holstein is a universal way so as to better improve the comprehensive benefits, as well as decline the inbreeding coefficient. However, the wrong parentage appeared frequently in this population than others due to not only the reasons in pure breeds, but more importantly, the lack of enough attention, which caused the lower accuracy of genetic parameter estimation and genetic evaluation in breeding systems. Single nucleotide polymorphism (SNP) panel in a certain population as a powerful tool for parentage assignment has been reported in numerous studies, especially in cattle. Therefore, the aim of this study was to build an SNP panel with sufficient power for parentage testing in the crossbred population of Simmental and Holstein in China.

Results: In the present study, combining direct sequencing method in polymerase chain reaction (PCR) products of deoxyribonucleic acid (DNA) pooling and matrix-assisted laser desorption/ionization time-offlight mass spectrometry (MALDI-TOF MS) genotyping method in individuals, a panel comprising 50 highly informative single nucleotide polymorphisms (SNPs) for parentage analysis was developed in a crossbred Chinese cattle population. The average minor allele frequency (MAF) was 0.43 and the cumulative exclusion probability for single-parent and both-parent inference met 0.99797 and 0.999999 , respectively. The maker-set was then used for parentage verification in a group of 81 trios with the likelihood-based parentage-assignment program of Cervus software. Compared with on-farm records, the results showed that this 50-SNP system could provide sufficient and reliable information for parentage testing with the parental mistakes for mother-offspring and sire-offspring being $8.6 \%$ and $18.5 \%$, respectively.

Conclusion: Knowledge of these results, we provided one set of low-cost and efficient SNPs for running paternity testing in the crossbred cattle population of Simmental and Holstein in China. Keywords: Parentage analysis, Single nucleotide polymorphism (SNP), Chinese crossbred cattle

\section{Background}

It is common knowledge that the success of genetic parameter estimation and genetic evaluation in national or international cattle breeding systems is directly affected by the accuracy of pedigrees. However, the wrong parentage appeared from time to time due to frequent use of artificial insemination (Al) and the lack of firmly maintaining reproduction records, and the inaccurate pedigree records [1]. Sanders et al. [2] had estimated the enormous influence of wrong and missing sire information on the reliability of estimated breeding values and genetic gain, especially those sires with small progeny size and traits with low heritability. An error rate by $10 \%$ in paternity determination would decline by $4.3 \%$ genetic gain per year [3]. Therefore, parentage testing, an essential tool for revising pedigree errors, has become an important element in both breeding practices and research. 
Recent advanced deoxyribonucleic acid (DNA) markers in single nucleotide polymorphism (SNP) research have become more and more popular and large numbers of dairy cattle were routinely genotyped for dense SNP chip or whole-genome re-sequenced for genomic selection [4] and genome-wide association studies [5], which also provided useful information for parentage assignment. Many studies have shown that significant progress in the utility of SNPs in cattle pedigree tests and some of them have presented preliminary panels in parentage testing, for example, in Israeli cattle breeds [6], in East Asian Korean Hanwoo and Japanese Wagyu [7], in American Angus beef cattle [8], as well as numbers of cattle breeds in Ireland [9]. In addition, as the information contained in an SNP-set may significantly be varied between different populations, it is necessary to develop an SNP panel with sufficient power to identify individuals and their parents in certain populations [7].

In the past decades, crossbreeding has been extensively practiced for improving milk production, milk composition, fertility, as well as calving ease worldwide [10-13]. In China, the widespread crossbreeding between Simmental and Holstein is a universal way so as to better improve the comprehensive benefits, as well as decline the inbreeding coefficient $[14,15]$. However, the mistakes of parentage occurred more frequently in crossbreed populations, not the least because such reasons appear in pure one but the pedigree registration not pay enough attention. In addition, there is no study to build one parentage testing system for the Chinese crossbred population, specifically. The aim of the present work was to select a set of SNPs with effective information and to estimate the potential utility of these markers for parentage testing in a Chinese crossbred population used two breeds, Simmental and Holstein, and provided one potential panel of SNPs for farmers and researchers running DNA analyzes for assignment of paternity purposes in that of the crossbred population in China.

\section{Results}

\section{SNP markers filtration}

In the present study, a total of 269 SNPs were pre-selected from previous reports and chip data of Chinese Holstein cattle, the direct sequencing of pooled DNA was chosen for evaluating SNP heterozygosity, and finally, 59 markers with high information were determined. Then, the MALDI-TOF MS method was used for genotyping the 59 SNPs in all the 168 cattle. Among the 59 SNPs, 9 were found to possess a call rate lower than $85 \%$ or being departed from the Hardy-Weinberg equilibrium and were deleted in further analysis. These 50 SNPs distributed on 27 autosomes (Fig. 1a), with an average call rate of $97.01 \%$ (8160/8400). The MAF ranged from 0.27 to 0.50 , with an average value of 0.43 in the total SNPs (Fig. 1b). The percentage of SNPs with a MAF between 0.45 and 0.50 was $46 \%$, and $74 \%$ of SNPs had a MAF ranging from 0.40 to 0.45 . In addition, the average $H_{O}$ of the remaining SNP loci was $0.497, \mathrm{H}_{E}$ was 0.484 , PIC was 0.366 (An additional file shows this in more detail [see Additional file 1]). The above results suggested that these 50 markers were highly informative and could be used as good genetic markers for parentage analysis in our crossbred population. 


\section{Parentage analysis with selected 50 SNPs and validation the effectiveness of assignment}

We used the 50 markers to construct parentage testing, and the cumulative probability of exclusion (CPE) for one-parent exclusion case and both-parent exclusion case was estimated to be 0.997974 and 0.999999 , respectively. The set of 50 SNPs was further used for verification of parentage testing in the population of 168 individuals. The results of parentage inference analyses for the 81 calves were summarized in Table 1. Of the 81 offspring-mother relationships, 79 had the confidence $>85 \%$, including 61 with confidence $\geq 95 \%$. For paternity inference, 66 calves had the confidence $\geq 95 \%$, with a ratio of $81.5 \%$, and 76 individuals had a confidence over $85 \%$. In detail, 7 calves with a confidence level lower than $85 \%$, and whose inferred parents were not the same as those recorded under the confidence beyond $85 \%$, were considered as error paternity record in this study. In total, there were 18 calves whose inferred parents were incompatible with the putative ones, among which 4 had both sire and dam pedigree errors. In addition, the paternity mistakes of sire-offspring and mother-offspring were $18.5 \%(15 / 81)$ and $8.6 \%$ (7/81), separately, which indicated the higher mistakes of paternity in sire-offspring than that of motheroffspring in this study.

Table 1

Maternity and paternity inference results

\begin{tabular}{|lllllll|}
\hline $\begin{array}{l}\text { Confidence } \\
\text { level }\end{array}$ & \multicolumn{2}{l}{ Maternity inference } & \multicolumn{3}{l|}{ Paternity inference } \\
\hline & Calves & Compatible & Incompatible & Calves & Compatible & Incompatible \\
\hline$\geq 95 \%$ & $61(75.3 \%)$ & 58 & 3 & $66(81.5 \%)$ & 59 & 7 \\
\hline $85 \% \varangle 95 \%$ & $18(22.2 \%)$ & 16 & 2 & $10(12.3 \%)$ & 7 & 3 \\
$<85 \%$ & $2(2.5 \%)$ & 0 & 2 & $5(4.9 \%)$ & 0 & 5 \\
$\begin{array}{l}\text { Incorrect } \\
\text { rate }\end{array}$ & & & $8.6 \%$ & & & $18.5 \%$ \\
\hline
\end{tabular}

\section{Discussion}

As a cost and time-effective design, pooling DNA has been used in many kinds of research, such as, detecting SNP [16], estimating allele frequencies [17], QTL mapping [18], as well as genome association scan [19]. In the present study, the pooled DNA was chosen for evaluating SNP heterozygosity, and finally, 59 markers were determined and selected from 269 candidates. According to the results of genotyping data in individuals, it demonstrated that the pooling DNA was a successful and effective way to identify markers with high information for parentage testing in a given population.

The method of MALDI-TOF MS was used to obtain the genotypes of 59 SNPs for individuals in the current study. For assessing the accuracy of this platform, 10 samples were repeated once and 936 data 
were gathered, out of which only one pair of a duplicate sample in one SNP data was not identical (data not shown), showing a genotyping error rate of 0.002 in MALDI-TOF MS genotyping method, the similar SNP genotyping error was reported by Heaton et al. [20] in sheep. In addition, according to Cooper et al. [21], the call rate of the animal was also related to genotyping accuracy, the individual call rate ranged from $96-99 \%$ in our study. All the above results revealed that the genotyping data got in the present study was validated, and the method of MALDI-TOF MS could be a stable platform for genotyping those SNPs $[22,23]$.

Given the effects of SNPs of call rate and Hardy-Weinberg equilibrium on parentage analysis in our population, 9 markers with a call rate lower than $85 \%$ or departed from Hardy-Weinberg equilibrium were detected and deleted in further analysis. Finally, we selected the panel of 50 informative SNPs with an average MAF value of $0.43, \mathrm{H}_{0}$ value of $0.497, \mathrm{H}_{E}$ value of 0.484 , and PIC value of 0.366 , which indicated that these SNPs have strong power for assignment of paternity in this crossbred cattle population. Generally, the cumulative probability of exclusion (CPE) for one-parent exclusion case and both-parent exclusion case was estimated to be 0.997974 and 0.999999 , that was consistent with the study of Zhang et al. [24] who reported 50 highly informative SNP markers for paternity testing in pure Simmental cattle population and suggested the CPE of $35-50$ SNPs could $>99 \%$ if selecting those highly heterozygous. In other earlier research, Herráeza et al. [6] documented that the exclusion power of 43 SNPs could exceed 98\% in Galloway cattle. The International Committee for Animal Recording (ICAR) has also recommended a cattle consensus panel of 100 core SNPs for genetic identification and parentage analysis [25]. However, few SNPs used in the present study were same as the above studies. The number one reason why those SNPs for paternity testing significantly vary between different populations is because core markers closely linked with heterozygosity and call rate of SNPs in the test population. Generally, more and more findings have reported the SNP panels for parentage verification in different cattle population, for instance, the Red Sindhi cattle in Brazil [26], the Brahman cattle in Costa Rica [27], the Angus Beef Cattle in United States of America [8], as well as the Holstein population in Mexico [28], so it is necessary to develop an SNP panel with sufficient power to identify individuals and their parents in certain populations [7]. Therefore, our work in the current study made up for the shortcomings of non-specific paternity identification markers for the crossbred population of Simmental and Holstein in China.

The set of 50 SNP markers was further used for verification of parentage testing in the population of 168 individuals. In the present study, 7 calves with a confidence level lower than $85 \%$ were considered as error paternity records. The reason for this was that their real mother or father was not sampled in this experiment, and there was a close relationship between two Simmental bulls i.e. one sire was the uncle of the other. What's more, 18 calves whose inferred parents were incompatible with the putative ones. Parental information of these 18 individuals was reconfirmed according to the on-farm records. The reasons for paternity mistakes were analyzed using both birth and calving data, as well as insemination records. Six calves had incorrect ear tags due to which their parent tracing was puzzled. In this farm, ear tagging of freshly born calves is practiced once a week not immediately after birth which caused paternity error in some calves. Three individuals were misidentified due to incorrect recording the semen 
or the cow label by Al technicians, and incorrect paternity recording for the other 8 calves were due to multiple inseminations using different sires. In Israel, multiple inseminations could explain at most $20 \%$ of the rejected paternity [1], however, in the present population, more than half of the paternity mistakes were due to this reason. Thence, the comparison results confirmed that pedigree inferred from the developed SNP-panel was correct, which absolutely showed the effective and powerful identification of this SNP paternity testing system. At the same time, combining the on-farm and genotypic data for paternity analysis is an effective option [29].

In the current study, according to the on-farm data, it can be confirmed that there were paternity mistakes of sire-offspring in $18.5 \%$ and mother-offspring in $8.6 \%$, which were in line with the reports by Chu et al. [30] and Guo et al. [31] who found the higher paternity mistakes in paternity inference than that of in maternity inference in Chinese Holstein population, and the reason of the multiple inseminations using different bulls may account for those results. Many countries had also reported the paternity error in cattle. In the United Kingdom (UK), Visscher et al. [32] reported a total paternity error rate of $10 \%$ in the dairy population. Similarly, 7\% paternity error was found in the Angeln dairy cattle population of German [2]. In Kenya, the sire misidentification rate was even over $50 \%$ in Boran cattle [33], which was much higher than the results of the present study. Those results suggest that the validation for parenting information is completely required in the cattle industry, efforts should be made to improve the accuracy of pedigree records. Therefore, parentage testing as an essential tool for correcting pedigrees are extremely important for both breeding and practice. Overall, our results showed that the set of 50 SNPs could be a practical tool for correcting pedigrees in the crossbred population of Simmental and Holstein in China.

\section{Conclusions}

In the present study, combining the previous reports and the chip data from Chinese Holstein population, and the result of direct sequencing of PCR products of pooled DNA and genotype data generated by MALDI-TOF MS method in individuals, a set consisting of 50 highly informative SNPs for parentage analysis was developed. In addition, the panel comprising 50 SNPs with an average MAF 0.43 for parentage analysis could reach 0.99797 and 0.999999 in CPE for single-parent and both-parent inference in present Chinese cattle population, respectively. And responding to on-farm records, the results of further validation showed that the 50-SNP system could provide sufficient and reliable information for parentage testing with the parental mistakes from mother-offspring and sire-offspring were $8.6 \%$ and $18.5 \%$, respectively. Given acknowledge of the above results, we provided one low-cost and efficient method of SNP assays for running paternity testing in crossbred cattle population of Simmental and Holstein in China.

\section{Methods}

\section{Animals and DNA extraction}


The use of the animals was in agreement with the Committee on Ethics of Animal Experimentation from the China Agricultural University. Seventy-five family trios, including calves and their registered parents, raised in Anshan Hengli Dairy Farm, China, were used in the present study. Those were: 1) 12 bulls from 2 breeds, 4 Chinese Holstein bulls and 8 Chinese Simmental bulls; 2) 75 Chinese Holstein cows; 3) 81 progeny with 3 half-sibs and 3 full-sibs, out of which 38 were crossbred from Simmental, and the other 43 were pure Holstein. In total, 168 cattle were used in the study. The cattle were housed in the opening cowshed and fed with the total mixed ration which was made up according to the Chinese Feeding Standard for cow cattle (NY/T 34-2004), while the water was accessible at all the time. Blood samples $(10 \mathrm{~mL})$ of calves and cows were harvested from the tail vein into anticoagulant tubes with EDTA-2K.

The genomic DNA was extracted from frozen semen of bulls, and from peripheral blood samples of cows and their calves using the DNA extraction kit (DP318, Tiangen Biotech Co., Beijing, China) according to the manufacturer's instructions. DNA content was measured with NanoDrop 2000 and adjusted to a final concentration of $50 \mathrm{ng} / \mu \mathrm{L}$. To facilitate searching and evaluation of SNPs, a DNA pool was constructed with an equal quantity of DNA from 30 unrelated individuals.

\section{Selection of SNP markers}

SNP markers were pre-selected from previous reports $[34,35]$ and from chip data with Illumina BovineHD BeadChip in Chinese Holstein cattle [31]. The selection criteria were: 1) the reported minor allele frequency (MAF) larger than 0.3, and 2) the genetic distance of markers in the same chromosome should be greater than $5 \mathrm{cM}$. Then, SNPs were re-selected according to their heterozygosity that was predicted by the direct sequencing in polymerase chain reaction (PCR) product of pooling DNA according to the previous study [36]. Only SNPs with an allele peak height ratio over 1:3 were considered to be highly heterozygosis and were reserved preferentially. Finally, 59 promising highly polymorphic SNP markers were determined.

\section{Genotype detection and Parentage analysis}

The matrix-assisted laser desorption/ionization time-of-flight mass spectrometry (MALDI-TOF MS) method was performed for genotyping the 59 markers of 168 individuals. Primers for multiple assays were designed using Assay Design Software (version 3.1). For accuracy control, it arranged 10 pairs of duplicate samples. Basic genetic parameters, including MAF, observed heterozygosity $\left(\mathrm{H}_{0}\right)$, expected heterozygosity $\left(\mathrm{H}_{E}\right)$, and polymorphic information content (PIC), for each SNP and paternity index were calculated with Cervus 3.0 software [24]. And parentage analysis was carried out using the likelihood method.

\section{Abbreviations}

\section{Al}

artificial insemination; DNA:deoxyribonucleic acid; SNP:single nucleotide polymorphism; MAF:minor allele frequency; PCR:polymerase chain reaction; MALDI-TOF MS:matrix-assisted laser desorption/ionisation time of flight mass spectrometry; $\mathrm{H}_{\mathrm{O}}$ :observed heterozygosity; $\mathrm{H}_{\mathrm{E}}$ :expected heterozygosity; 
PIC:polymorphic information content; QTL:quantitative trait locus; CPE:cumulative probability of exclusion; ICAR:International Committee for Animal Recording; UK:United Kingdom.

\section{Declarations}

\section{Ethics approval and consent to participate}

The use of the animals was in agreement with the Committee on Ethics of Animal Experimentation from the China Agricultural University. The experiment was performed according to regulations and guidelines established by this committee. The use of the animals has obtained written informed consent in this study from the owner of the animals.

\section{Consent for publication}

Not applicable.

\section{Availability of data and materials}

Yachun Wang is a member of the editorial board (Associate Editor) of this journal.

\section{Competing interests}

The authors declare that they have no competing interests.

\section{Funding}

This work was supported by the China Agriculture Research System (CARS-36), and the Program for Changjiang Scholar and Innovation Research Team in University (IRT_15R62). The China Agriculture Research System was used to pay for materials and sequencing for the PCR analyses. The Program for Changjiang Scholar and Innovation Research Team in University was used to pay for the analyses of the SNP genotype of individuals.

\section{Authors' contributions}

YCW and QC designed the experiment and supervised the project. LRH wrote the manuscript of the paper. DL carried out the data analysis and wrote the initial manuscript. $L Z$ and $Y Y$ helped to conduct the lab work. YZ and SLZ provided chip data. UT provided English editing. ZQX and SYH helped to collect the blood samples. LL and WHS provided the experimental animals and on-farm information. All authors read, edited, and approved the last manuscript.

\section{Acknowledgments}

Authors are grateful to Geoffrey Kirton, Visiting Professor in College of Animal Science and Technology, China Agricultural University for his serious proofreading of this manuscript. 


\section{Authors' information}

Lirong Hu${ }^{1}$, lironghu92@gmail.com; Dong Li ${ }^{12}$, 251735626@qq.com; Qin Chu ${ }^{3}$, chuqinsd@163.com; Yachun Wang ${ }^{1}$, wangyachun@cau.edu.cn; Lei Zhou ${ }^{1}$, zhoulei1112@126.com; Ying Yu ${ }^{1}$, yuying@cau.edu.cn; Yuan Zhang ${ }^{1}$, changy@cau.edu.cn; Shengli Zhang ${ }^{1}$, zhang62733697@163.com; Tahir Usman ${ }^{14}$, tahirusman@awkum.edu.pk; Zhenquan Xie ${ }^{5}$, Inhengli@188.com; Shiyu Hou ${ }^{5}$, 159599808@qq.com; Lin Liu6, liulin@bdcc.com.cn; Wanhai Shi6 ${ }^{6}$ 13501063706@163.com.

${ }^{1}$ Key Laboratory of Animal Genetics, Breeding and Reproduction, MARA; National Engineering Laboratory for Animal Breeding; Beijing Engineering Technology Research Center of Raw Milk Quality and Safety Control; College of Animal Science and Technology, China Agricultural University, Beijing 100193, China

${ }^{2}$ Beijing Xiangzhong Biotechnology Co. LTD, Beijing 100080, China

${ }^{3}$ Institute of Animal Husbandry and Veterinary Medicine, Beijing Academy of Agriculture and Forestry Sciences, Beijing 100097, P.R. China

${ }^{4}$ College of Veterinary Sciences and Animal Husbandry, Abdul Wali Khan University, Mardan 23200, Pakistan

${ }^{5}$ Anshan Hengli Dairy Farm, Anshan, Liaoning 114200, P.R. China

${ }^{6}$ Beijing Dairy Cattle Center, Beijing 100192, P.R. China

\section{References}

1. Weller JI, Feldmesser E, Golik M, Tager-Cohen I, Domochovsky R, Alus O, et al. Factors affecting incorrect paternity assignment in the Israeli Holstein population. J Dairy Sci. 2004;87(8):2627-40.

2. Sanders K, Bennewitz J, Kalm E. Wrong and missing sire information affects genetic gain in the Angeln dairy cattle population. J Dairy Sci. 2006;89(1):315-21.

3. Israel C, Weller Jl. Effect of misidentification on genetic gain and estimation of breeding value in dairy cattle populations. J Dairy Sci. 2000;83(1):181-7.

4. Wiggans GR, Cole JB, Hubbard SM, Sonstegard TS. Genomic Selection in Dairy Cattle: The USDA Experience. Annu Rev Anim Biosci. 2017;5(1):309-27.

5. Calderón-Chagoya R, Hernandez-Medrano JH, Ruiz-López FJ, Garcia-Ruiz A, Vega-Murillo VE, Montano-Bermudez M, et al. Genome-Wide Association Studies for Methane Production in Dairy Cattle. Genes. 2019;10(12):995.

6. Herraez DL, Schäfer H, Mosner J, Fries HR, Wink M. Comparison of microsatellite and single nucleotide polymorphism markers for the genetic analysis of a Galloway cattle population. Zeitschrift für Naturforschung C. 2005;60(7-8):737-643. 
7. Strucken EM, Gudex B, Ferdosi MH, Lee HK, Song KD, Gibson JP, et al. Performance of different SNP panels for parentage testing in two East Asian cattle breeds. Anim Genet. 2014;45(4):572-5.

8. Williams J, Reecy J, Honeyman M. Use of parentage testing in the ISU McNay Angus beef battle hHerd. Farm Progress Reports. 2018;2017(1):22-3.

9. McClure MC, McCarthy J, Flynn P, McClure JC, Dair E, O'Connell DK, et al. SNP data quality control in a national beef and dairy cattle system and highly accurate SNP based parentage verification and identification. Front Genet. 2018;9:84.

10. de Haas Y, Smolders EA, Hoorneman JN, Nauta WJ, Veerkamp RF. Suitability of cross-bred cows for organic farms based on cross-breeding effects on production and functional traits. Animal. 2013;7(4):655-65.

11. Hazel AR, Heins BJ, Hansen LB. Fertility, survival, and conformation of Montbéliarde $\times$ Holstein and Viking Red $\times$ Holstein crossbred cows compared with pure Holstein cows during first lactation in 8 commercial dairy herds. J Dairy Sci. 2017;100(11):9447-58.

12. Hazel AR, Heins BJ, Hansen LB. Production and calving traits of Montbéliarde $\times$ Holstein and Viking Red $\times$ Holstein cows compared with pure Holstein cows during first lactation in 8 commercial dairy herds. J Dairy Sci. 2017;100(5):4139-49.

13. Maurmayr A, Pegolo S, Malchiodi F, Bittante G, Cecchinato A. Milk protein composition in purebred Holsteins and in first/second-generation crossbred cows from Swedish Red, Montbeliarde and Brown Swiss bulls. Animals. 2018;12(10):2214-20.

14. Wang ZG, Chang Y, Qiu XT, Sun ZH, Zhang BS, Wang YC. Analysis of Efficiency of Crossbred Cows between German Simmental and Holstein. China Dairy Cattle. 2017(12):26-30.

15. Chen N, Chen HL, Zhang YF, Sheng M, Hou FQ, Feng XL, et al. Meat Production Performance and Meat Quality of Holstein Cows and German Simmental Hybrid Offspring. Journal of Henan Agricultural Sciences. 2017;46(1):136-9.

16. Jiao S, Chu Q, Wang Y, Xie Z, Hou S, Liu A, et al. Identification of the causative gene for Simmental arachnomelia syndrome using a network-based disease gene prioritization approach. PLoS One. 2013;8(5):e64468.

17. Ozerov M, Vasemägi A, Wennevik V, Niemelä E, Prusov S, Kent M, et al. Cost-effective genome-wide estimation of allele frequencies from pooled DNA in Atlantic salmon (Salmo salarL.). BMC Genom. 2013;14(1):12.

18. Strillacci MG, Frigo E, Schiavini F, Samoré AB, Canavesi F, Vevey M, et al. Genome-wide association study for somatic cell score in Valdostana Red Pied cattle breed using pooled DNA. BMC Genet. 2014;15(1):106.

19. Fowdar JY, Grealy R, Lu Y, Griffiths LR. A genome-wide association study of essential hypertension in an Australian population using a DNA pooling approach. Mol Genet Genomics. 2017;292(2):307-24.

20. Heaton MP, Leymaster KA, Kalbfleisch TS, Kijas JW, Clarke SM, McEwan J, et al. SNPs for parentage testing and traceability in globally diverse breeds of sheep. PLoS One. 2014;9(4):e94851. 
21. Cooper TA, Wiggans GR, VanRaden PM. Short communication: relationship of call rate and accuracy of single nucleotide polymorphism genotypes in dairy cattle. J Dairy Sci. 2013;96(5):3336-9.

22. Li R, Xiao D, Yang J, Sun S, Kaplan S, Li Z, et al. Identification and Characterization of Clostridium difficile Sequence Type 37 Genotype by Matrix-Assisted Laser Desorption Ionization-Time of Flight Mass Spectrometry. J Clin Microbiol. 2018, 56(5).

23. Megha K, Sharma M, Gupta A, Sehgal R, Khurana S. Protein profiling of Acanthamoeba species using MALDI-TOF MS for specific identification of Acanthamoeba genotype. Parasitol Res. 2018;117(3):729-36.

24. Zhang T, Guo L, Shi M, Xu L, Chen Y, Zhang L, et al. Selection and effectiveness of informative SNPs for paternity in Chinese Simmental cattle based on a high-density SNP array. Gene. 2018;673:211-6.

25. McClure MC, McCarthy J, Flynn P, Weld R, Keane M, O'connel K, et al. SNP selection for nationwide parentage verification and identification in beef and dairy cattle. ICAR Tech Ser. 2015.

26. Panetto JCdC, Machado MA, da Silva MVGB, Barbosa RS, dos Santos GG, Leite RdMH, et al. Parentage assignment using SNP markers, inbreeding and population size for the Brazilian Red Sindhi cattle. Livestock Science. 2017;204:33-8.

27. Campos J, Vargas B, Camacho J, Cruz A. Identity and parentage testing in registered Brahman cattle of Costa Rica. Agronomía Costarricense. 2018;42(1):49-62.

28. García-Ruiz A, Wiggans GR, Ruiz-López FJ. Pedigree verification and parentage assignment using genomic information in the Mexican Holstein population. J Dairy Sci. 2019;102(2):1806-10.

29. Fisher PJ, Malthus B, Walker MC, Corbett G, Spelman RJ. The number of single nucleotide polymorphisms and on-farm data required for whole-herd parentage testing in dairy cattle herds. $J$ Dairy Sci. 2009;92(1):369-74.

30. Chu Q, Zhang Y, Sun DX, Yu Y, Wang YC, Zhang Y. Pedigree verification of Holstein cows and analysis of factors affecting incorrect paternity based on microsatellite markers. Chinese Journal of Animal Veterinary Sciences. 2011;42:163-8.

31. Guo G, Zhou L, Liu L, Li D, Zhang SL, Liu JF, et al. Parentage Inference with Single Nucleotide Polymorphism Markers in the Chinese Holstein in Beijing. Chinese Journal of Animal Veterinary Sciences. 2012;43(01):44-9.

32. Visscher PM, Woolliams JA, Smith D, Williams JL. Estimation of pedigree errors in the UK dairy population using microsatellite markers and the impact on selection. J Dairy Sci. 2002;85(9):236875.

33. Kios D, van Marle-Koster E, Visser C. Application of DNA markers in parentage verification of Boran cattle in Kenya. Trop Anim Health Prod. 2012;44(3):471-6.

34. Heaton MP, Harhay GP, Bennett GL, Stone RT, Grosse WM, Casas E, et al. Selection and use of SNP markers for animal identification and paternity analysis in U.S. beef cattle. Mamm Genome. 2002;13(5):272-81.

35. Werner FA, Durstewitz G, Habermann FA, Thaller G, Kramer W, Kollers S, et al. Detection and characterization of SNPs useful for identity control and parentage testing in major European dairy 
breeds. Anim Genet. 2004;35(1):44-9.

36. Hu L, Ma Y, Liu L, Kang L, Brito LF, Wang D, et al. Detection of functional polymorphisms in the hsp70 gene and association with cold stress response in Inner-Mongolia Sanhe cattle. Cell Stress Chaperones. 2019;24(2):409-18.

\section{Figures}

a

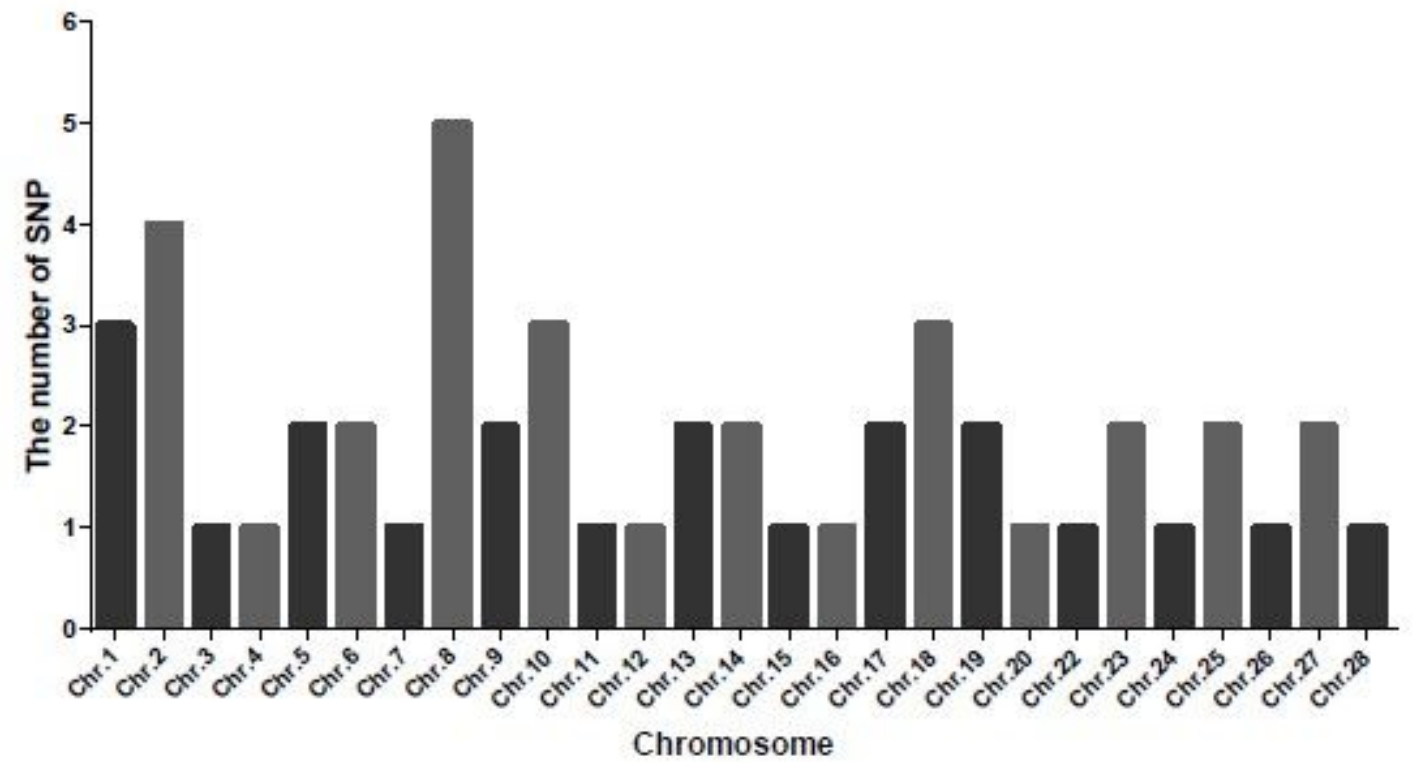

b

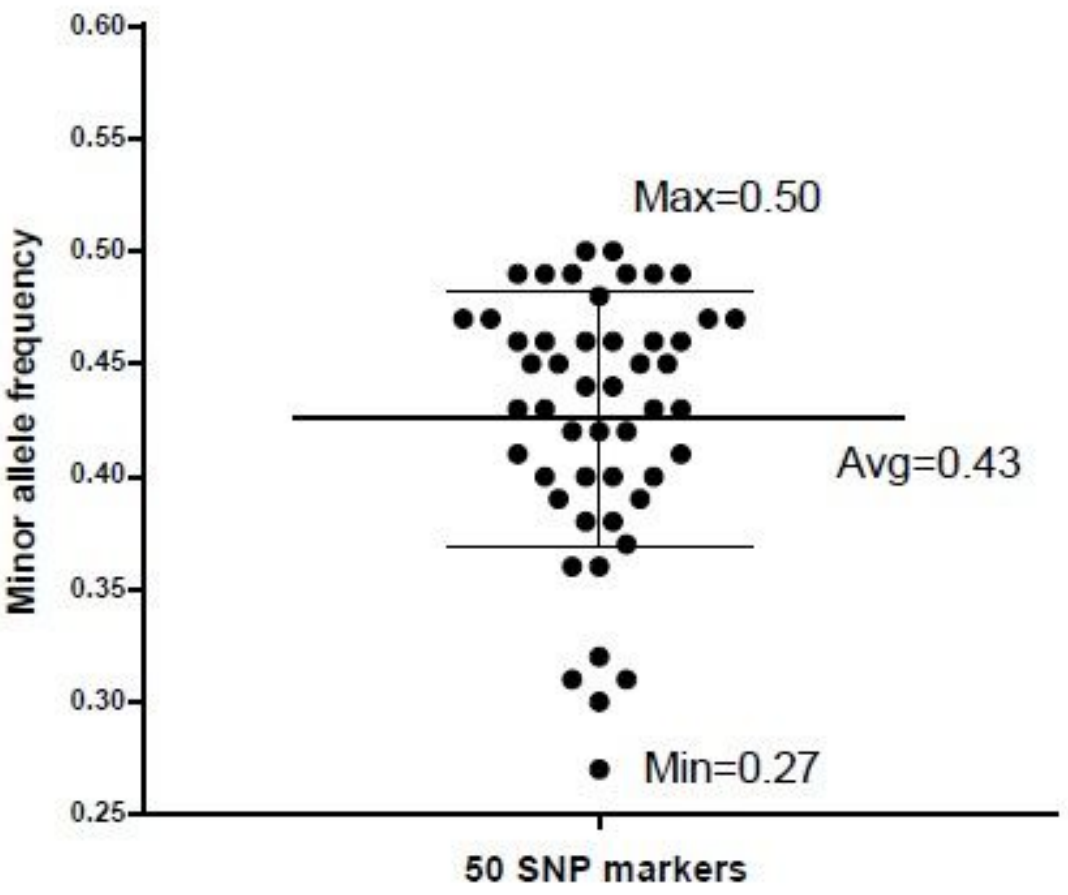

Figure 1

The chromosome distribution (a) and MAF distribution (b) of the 50 SNP markers. 


\section{Supplementary Files}

This is a list of supplementary files associated with this preprint. Click to download.

- Additionalfile1TableS1.docx 